\title{
Nanotopographic Control of Neuronal Polarity
}

\author{
Aldo Ferrari, ${ }^{*+, \perp}$ Marco Cecchini, ${ }^{*,+}$ Akshay Dhawan, ${ }^{\ddagger}$ Silvestro Micera, ${ }^{\prime}$ Ilaria Tonazzini, ${ }^{\dagger}$ Ripalta Stabile, ${ }^{\S}$ \\ Dario Pisignano, ${ }^{\S}$ and Fabio Beltram ${ }^{+}$ \\ ${ }^{\dagger}$ NEST, Istituto Nanoscienze - CNR and Scuola Normale Superiore, Piazza San Silvestro, 12 I-56126 Pisa, Italy \\ ${ }^{\ddagger}$ School of Electrical and Computer Engineering, Cornell University, Ithaca, New York 14853, United States \\ ${ }^{\S} \mathrm{NNL}$, Istituto Nanoscienze - CNR, IIT Center for Biomolecular Nanotechnologies, and Dipartimento di Ingegneria dell'Innovazione, \\ Università del Salento, via Arnesano, 73100, Lecce, Italy \\ "ARTS Lab, Scuola Superiore Sant'Anna and Institute for Automation, Swiss Federal Institute of Technology Zurich, 8006 Zurich, \\ Switzerland
}

Supporting Information

ABSTRACT: We employ simple geometrical rules to design a set of nanotopographies able to interfere with focal adhesion establishment during neuronal differentiation. Exploiting nanoimprint lithography techniques on cyclic-olefin-copolymer films, we demonstrate that by varying a single topographical parameter the orientation and maturation of focal adhesions can be finely modulated yielding independent control over the final number and the outgrowth direction of neurites. Taken

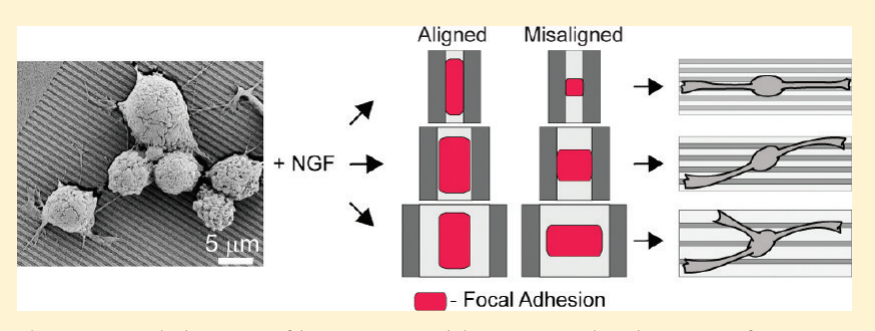
together, this report provides a novel and promising approach to the rational design of biocompatible textured substrates for tissue engineering applications.

KEYWORDS: Cell polarity, neuron, focal adhesions, contact guidance, topography

$\mathrm{T}$ he mammalian neuronal network provides the best example of a highly polarized tissue whose functions depend on the underlying cell polarity. ${ }^{1}$ Neuronal differentiation is governed by molecular stimuli, acting over long distances, and by physical signals locally retrieved through contact guidance (i.e., cell-shape adaptation to the local extracellular environment). ${ }^{2}$ Young neurons integrate this information and produce long cellular extensions (the neurites) in a process called neuronal polarity establishment. ${ }^{1}$ Specifically, the initiation and engorgement of a nascent neurite are critically controlled by the establishment and maturation of focal adhesions (FAs), the integrin-based cellular structures linking the cell to the underlying substrate, at the tip of lamellipodia and filopodia. ${ }^{3}$ In a second step, the developing neurites test the surrounding environment searching the way toward their final targets in a process termed neurite pathfinding. ${ }^{4}$ Neuronal polarity establishment and neurite pathfinding are the most important processes to build a functional tissue during neuronal development and regeneration. ${ }^{1,5}$

The function of FAs goes far beyond the simple physical anchorage. ${ }^{6}$ Each of the adhesions initially established by a differentiating neuron undergoes a modular maturation and develops from an isotropic focal complex to a mature and anisotropic FA. ${ }^{7}$ Eventually, a mature FA can induce local actin polymerization and thus control the rearrangement of the cell shape. ${ }^{8}$ Maturation of FAs is critically modulated by the local physical and chemical properties of the host substrate, ${ }^{9-11}$ and therefore the cell gathers multiple information regarding substrate properties such as the mechanical rigidity, the density of adhesion points, their chemical identity and geometrical distribution, and the surface topography. ${ }^{12,13}$ Importantly, through the modulation of FA maturation, a specific extracellular configuration can define the cell fate and function. ${ }^{14,15}$

The advancement of nano- and microfabrication technologies allows the generation of biocompatible substrates bearing variable topography in the critical ranges affecting cellular functions. ${ }^{16,17}$ In particular, contact guidance and neurite pathfinding were investigated in PC12 cells contacting topographically modified materials such as silicon substrates. ${ }^{18,19}$ The synergic effect of topography and soluble neurotropic stimuli was also addressed by reports investigating neuronal differentiation on nanogratings and polymeric nanofibers. ${ }^{20,21}$ Altogether, these works provide evidence for the possibility to influence neuronal differentiation through the topographical modification of the surface. ${ }^{22}$ However, the design of scaffolds that selectively induce specific neuronal polarity states requires a deeper understating of the interplay between the involved cellular machineries and the local nanotopography.

In this direction we recently demonstrated that anisotropic topographies, nanogratings (alternating lines of grooves and ridges with submicrometer lateral dimension), select bipolar cells with two aligned neurites during nerve growth factor (NGF)induced PC12 differentiation. ${ }^{10}$ The selection is achieved through

Received: September 22, 2010

Revised: December 20, 2010 
a direct modulation of FA maturation and can be condensed into four basic steps. First, FAs are exclusively established on top of ridges and cannot bridge across grooves larger than $500 \mathrm{~nm}$. Second, FAs produced at the tip of aligned neurites (within $15^{\circ}$ to the direction of the nanograting) mature, whereas those produced at the tip of misaligned processes remain immature. Third, mature FAs persist longer, thus allowing the consolidation of the respective neurites. Finally, the selected polarity state is maintained through the polarization of the neurite initiation machinery. ${ }^{10}$

Here we exploit the interaction between FAs and topographic features to design, fabricate, and validate a set of original scaffolds made of cyclic olefin copolymer (COC) yielding precise control over neuronal polarity establishment and neurite pathfinding. These substrates are efficient in selectively enriching the population of specific neuronal polarity states, namely, bipolar cells with aligned neurites, bipolar cells with misaligned neurites, or multipolar cells with misaligned neurites.

Rational Design of Nanogratings. FAs generated by PC12 cells contacting flat substrates (between 12 and $24 \mathrm{~h}$ after NGF stimulation) are, on average, $1600 \mathrm{~nm}$ long and $900 \mathrm{~nm}$ wide (Supplementary Figure 1a,b, Supporting Information). Importantly, on nanogratings with $500 \mathrm{~nm}$ wide ridges and grooves, FA establishment is restricted to individual ridges. This topographical constraint affects FA maturation by demoting the length increase in misaligned FAs (between $15^{\circ}$ and $90^{\circ}$ to the main direction of the nanogratings), thus blocking their maturation, and by limiting the width increase in aligned FAs (within $15^{\circ}$ to the main direction of the nanogratings), while promoting their elongation. The net result of this cell-topography interaction is the selection of bipolar cells with two aligned neurites. ${ }^{10}$

Starting from these considerations, a set of nanogratings is produced, with constant groove size and depth (500 and $350 \mathrm{~nm}$, respectively) and increasing ridge width, ranging from $500 \mathrm{~nm}$ (smaller than the typical FA length and width) to $2000 \mathrm{~nm}$ (larger than the typical FA length and width). The rationale behind this design is that a gradual release of the topographic constrain would result in a modulation of neuronal polarity selection by allowing the maturation of misaligned FA (Supplementary Figure 1c, Supporting Information).

Material Characterization and Fabrication Process -. The nanogratings are transferred on COC foils using thermal nanoimprint lithography (NIL). To prepare the substrates, p-doped, silicon wafers are used as molds and undergo electron beam lithography (EBL) and reactive ion etching (RIE). After a layer of $250 \mathrm{~nm}$ thick poly(methyl methacrylate) (PMMA) is deposited, $\mathrm{EBL}$ is used to expose the grating pattern. Sulfur hexafluoride $\left(\mathrm{SF}_{6}\right)$-based RIE is then used to transfer the pattern to the silicon substrate. $^{23}$

Six molds are fabricated with ridge width of $500 \mathrm{~nm}$, depths of $350 \mathrm{~nm}$, and groove widths of $500 \mathrm{~nm}$ (nanograting “A”), $750 \mathrm{~nm}$ (“B”), $1000 \mathrm{~nm}$ (“C”), $1250 \mathrm{~nm}$ (“D”), $1500 \mathrm{~nm}$ (“E”), and 2000 $\mathrm{nm}$ ("F"), respectively (Supplementary Figure 2, Supporting Information). The molds are cleaned by rinsing with acetone and 2-propanol. After an oxygen plasma treatment, the molds are silanized by immersion in dimethyldichlorosilane for $15 \mathrm{~min}$ to facilitate the separation of the master and replica after NIL. The COC substrates are then placed on top of the mold and softened by raising the temperature up to $150^{\circ} \mathrm{C}$. A pressure of $50 \mathrm{bar}$ is then applied for $5 \mathrm{~min}$ before cooling down to $70{ }^{\circ} \mathrm{C}$, i.e., below the glass transition temperature of the copolymer $\left(T_{\mathrm{g}}=134^{\circ} \mathrm{C}\right)$. Finally the pressure is released and the mold is detached from the substrate with a scalpel.

\section{a}

\begin{tabular}{|c|c|c|}
\hline $\begin{array}{c}\text { Elastic Modulus } \\
\text { (MPa) }\end{array}$ & $\begin{array}{c}\text { Stress at break } \\
\text { (MPa) }\end{array}$ & $\begin{array}{c}\text { Strain at break } \\
(\%)\end{array}$ \\
\hline $2470 \pm 200$ & $58 \pm 6$ & $3.3 \pm 0.4$ \\
\hline
\end{tabular}

b

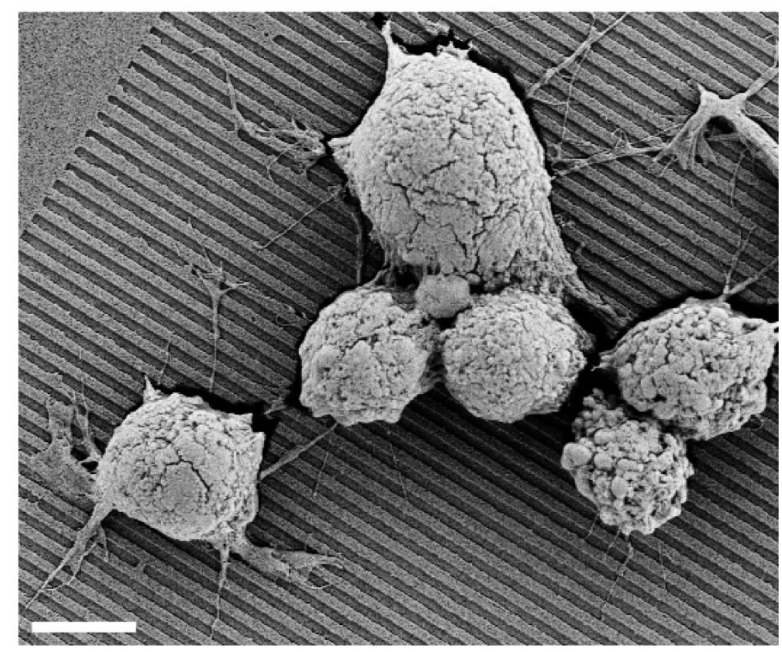

C

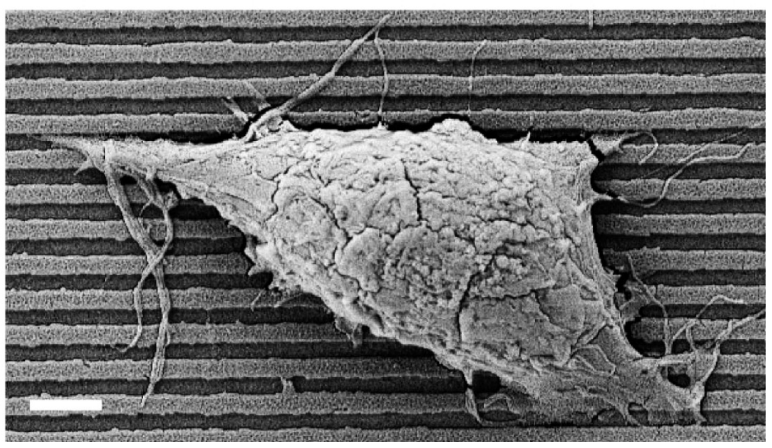

Figure 1. Topographically modified COC substrate for neuronal polarity selection. (a) Characterization of the mechanical stiffness of the substrate. Scanning electron micrographs of PC12 cells growing on nanogratings with $500 \mathrm{~nm}$ ridge width, (b) before and (c) $12 \mathrm{~h}$ after stimulation with NGF. Scale bars in (b) and (c) correspond to $5 \mu \mathrm{m}$.

The mechanical properties of the substrate are known to influence neuronal cell growth, contact guidance, and differentiation. ${ }^{24,25}$ In order to assess the mechanical stiffness of the COC nanogratings, the tensile stress, strain, and elastic modulus are calculated averaging the results obtained from the load elongation curves acquired from 15 different specimens. The results are reported in Figure 1a. Importantly, yielding was not observed, indicating that the substrates present high rigidity, in keeping with the measured values for the elastic E-modulus.

Finally, cell viability is tested by growing PC12 cells on nanogratings. PC12 cells are perfectly viable on all tested substrates and display no visible differentiation before stimulation with NGF (Figure 1b). Additionally, the geometry of the underlying topographical features is not altered by the interaction between cells and substrate, indicating that the stiffness of the substrate resists to the mechanical stress induced by cell-generated forces at the tip of developing neurites (Figure 1c). ${ }^{26}$

Effect of Nanogratings with Increasing Ridge Width on Neuronal Differentiation. Useful cellular models of mammalian 

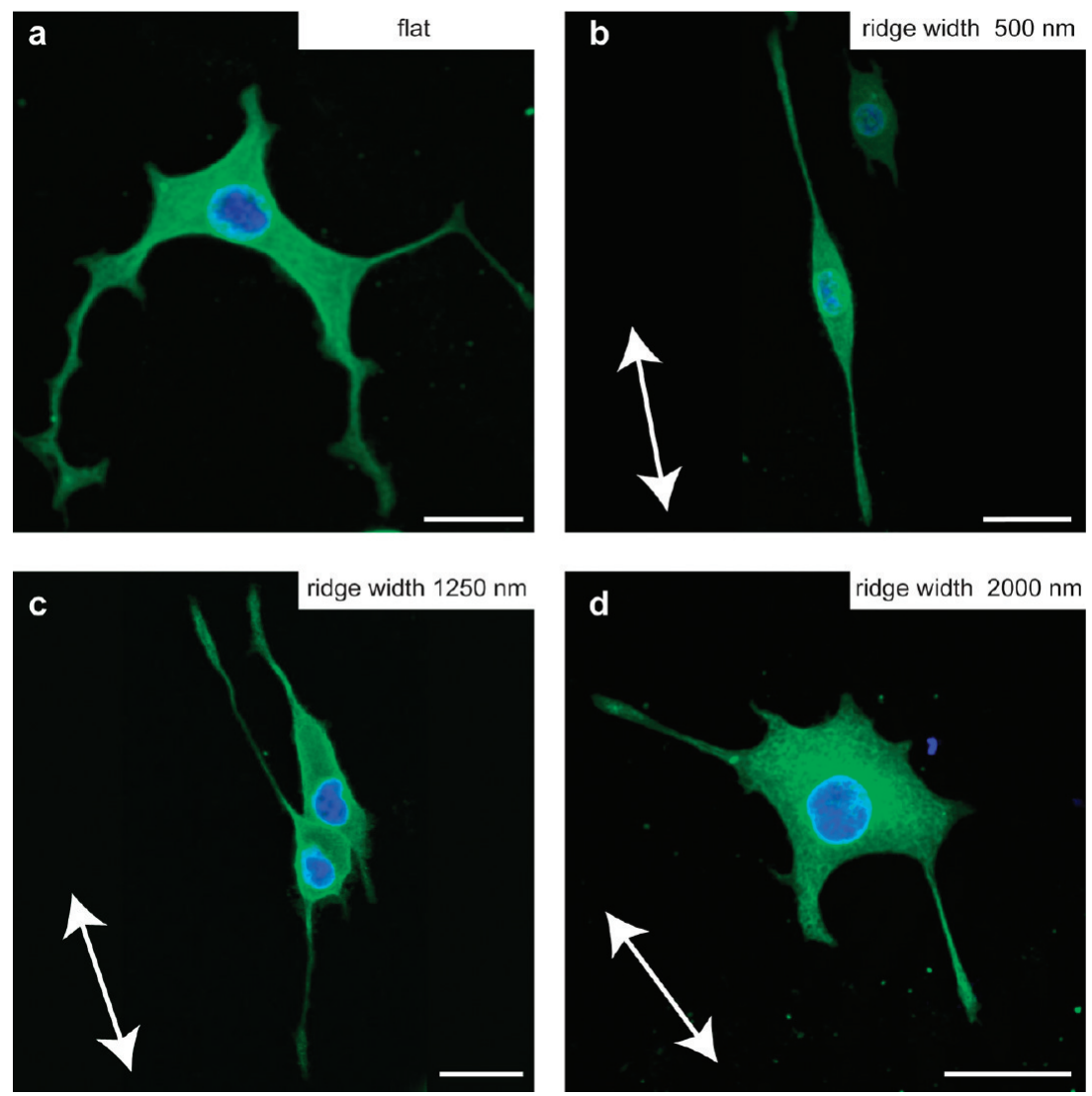

Figure 2. Neuronal differentiation of PC12 cells on nanogratings. Confocal images of representative PC12 cells on (a) flat substrates or on nanogratings with ridge width corresponding to (b) $500 \mathrm{~nm}$, (c) $1250 \mathrm{~nm}$ or (d) $2000 \mathrm{~nm}, 96 \mathrm{~h}$ after stimulation with NGF. Cells are stained with the nuclear marker DAPI (blue) and with the neuronal marker $\beta$-III-tubulin (green). The corresponding ridge width is reported in the upper right corner. Scale bars correspond to $20 \mu \mathrm{m}$. White arrows in each panel indicate the direction of the nanograting.

neuronal differentiation, such as PC12 cells, recapitulate in vitro all the relevant steps in polarity establishment and neurite pathfinding upon stimulation with neurotropic molecules such as $\mathrm{NGF}^{27}$ Bona fide neuronal differentiation is assessed revealing the expression of the neuronal differentiation marker $\beta$-III-tubulin which becomes evident already $12 \mathrm{~h}$ after stimulation (Supplementary Figure 3, Supporting Information). PC12 cells contacting flat or textured COC substrates generate long cellular protrusions within $96 \mathrm{~h}$ of treatment with NGF (Figure 2). Neurites generated by cells contacting flat COC substrates (Figure 2a) or nanogratings A (Figure 2b), D (Figure 2c), and F (Figure 2d) are stained to similar levels by the antibody against $\beta$-III-tubulin. Altogether, these results confirm that our COC substrates provide an optimal platform for the study of polarity establishment during neuronal differentiation of PC12 cells.

Angular Modulation of Focal Adhesion Maturation. In order to assess FA formation and maturation in PC12 cells differentiating on nanogratings, we induce the expression of paxillin-EGFP (Figure 3), a fluorescent mutant of paxillin which is readily included in focal complexes and FAs without affecting their maturation and functionality. ${ }^{28}$ The analysis of paxillinEGFP distribution confirms that the localization of FAs on nanogratings coincides with and is restricted to the position of the ridges on all tested nanogratings (Figure 3).

Paxillin-rich adhesions formed between 12 and $24 \mathrm{~h}$ after NGF stimulation are visibly aligned to the nanogratings on substrates presenting the smallest ridge width (500 and $750 \mathrm{~nm}$, Figure 3a,b).
Only few, small misaligned adhesions are detected in cells differentiating on these substrates (Figure 3b). Interestingly, a significant number of small, misaligned FAs are detected on nanogratings with ridge width of $1000 \mathrm{~nm}$ (Figure 3c). Misaligned FAs further increase, in number and size, on nanogratings with larger ridge width (1250 and $1500 \mathrm{~nm}$, Figure 3d,e) to the point that no preferential orientation is observed for FAs generated on nanogratings with ridge width of $2000 \mathrm{~nm}$ (Figure 3f).

In order to quantify the effect of the cell-to-topography interaction on FA maturation, we measure the size of FAs generated by PC12 cells differentiating on nanogratings. The topographical constraint produced by FA confinement on ridges results in a difference in size between FAs formed at the tip of aligned neurites and those generated at the tip of misaligned ones. The graph in Figure 4a depicts this measure as a ratio between the size of aligned and misaligned FAs for the different nanograting geometries. Aligned FAs generated by $\mathrm{PC} 12$ on nanogratings with small ridge width (A and B) are significantly larger than misaligned ones (aligned/misaligned size ratios $1.7 \pm 0.2$ and $1.9 \pm 0.2$ for FAs generated on nanograting $\mathrm{A}$ and $\mathrm{B}$, respectively). On nanogratings $\mathrm{C}$ the measured FA size ratio is slightly reduced $(1.4 \pm 0.1$, Figure $4 a)$, thus indicating a reduced difference between the size of aligned and misaligned FAs. Importantly on nanogratings with larger ridge width (D, E, and F; Figure 4a) the FA size ratio significantly decreases to values close to $1(1.1 \pm 0.2,1.2 \pm 0.2$, and $1.1 \pm 0.1$ for nanogratings $\mathrm{D}, \mathrm{E}$, and F, respectively). These results confirm that the angular modulation of FA maturation (generated by the topographical 

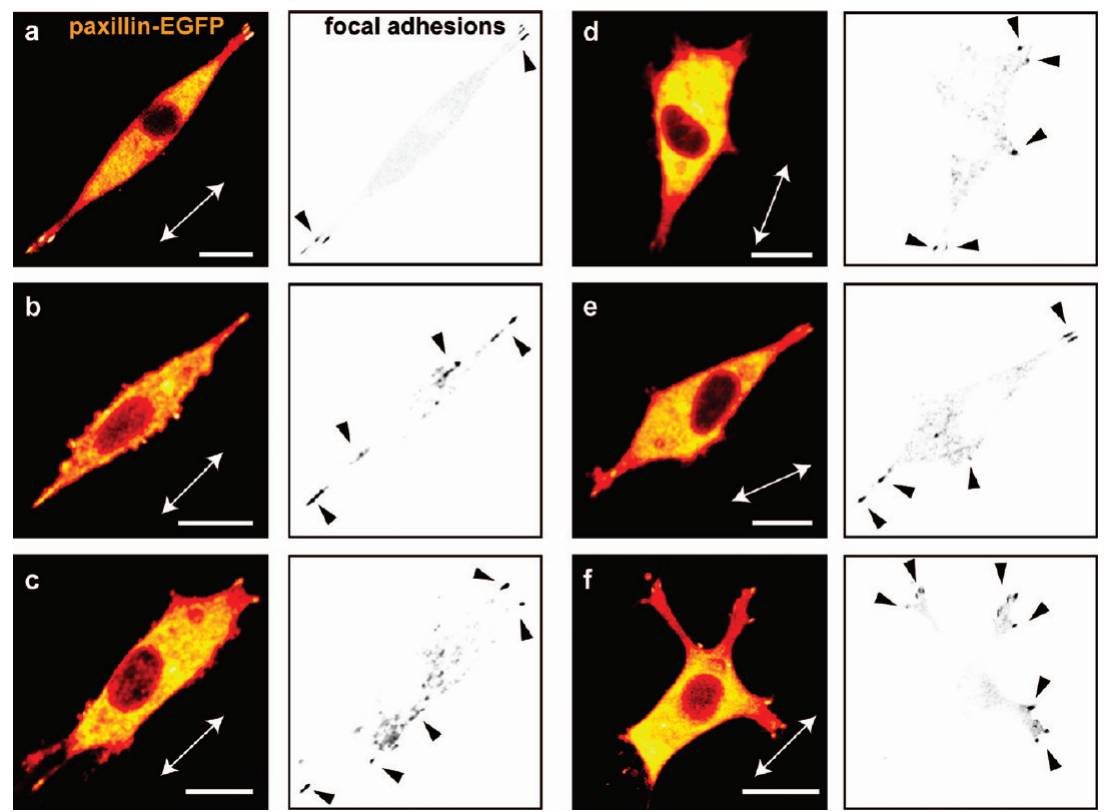

Figure 3. Focal adhesion establishment by stimulated PC12 contacting nanogratings with increasing ridge width. Distribution of paxillin-EGFP fluorescent signal (between 12 and $24 \mathrm{~h}$ after NGF stimulation) in PC12 cells differentiating on nanogratings with ridge width corresponding to (a) $500 \mathrm{~nm}$, (b) $750 \mathrm{~nm}$, (c) $1000 \mathrm{~nm}$, (d) $1250 \mathrm{~nm}$, (e) $1500 \mathrm{~nm}$ or (f) $2000 \mathrm{~nm}$. The maximum projections of confocal Z-stacks are reported in the left panel (red-hot look-up table; a-f). White arrows indicate the direction of the nanograting. Scale bars correspond to $10 \mu \mathrm{m}$. In the right panel, inverted fluorescent signal at the cell-substrate interface pinpoints the corresponding regions of paxillin-EGFP accumulation (black spots). Black arrowheads point to individual focal adhesions visible at the tip of developing neuronal processes.

a

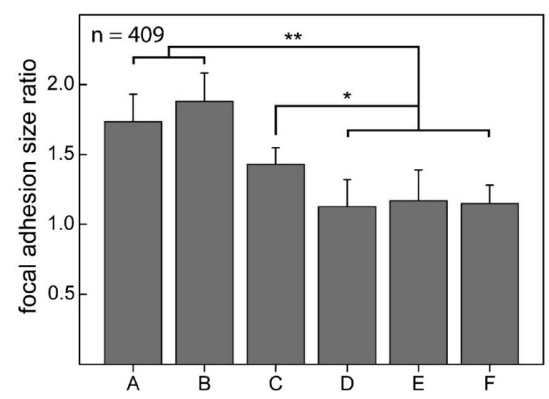

b

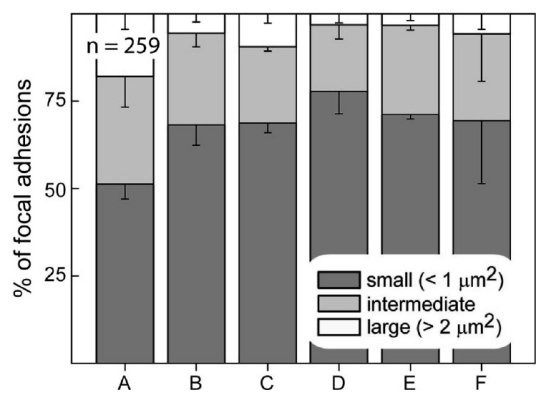

Figure 4. Modulation of FA maturation by nanogratings with increasing ridge width. (a) Average measured ratio between the size of aligned $\left(0-15^{\circ}\right)$ and misaligned $\left(15-90^{\circ}\right)$ adhesions established by PC12 after 12-24 h of NGF stimulation on nanogratings. Error bars correspond to the measured standard errors of the mean. Statistically significant differences between reported averages (as identified by black horizontal lines connecting two or more histograms) are reported by a single asterisk for $p<0.05$ and with two asterisks when $p<0.01$. (b) The stacked vertical histograms report the percentage of small ( $<1 \mu \mathrm{m}^{2}$, dark gray), intermediate (between 1 and $2 \mu \mathrm{m}^{2}$, gray), and large ( $>2 \mu \mathrm{m}^{2}$, white) FAs established by PC12 cells after $12-24 \mathrm{~h}$ of NGF stimulation on nanogratings. The number of analyzed elements is reported in the upper left corner of the graph. Error bars correspond to the measured standard errors of the mean.

constraint imposed by the ridge width) is effective on nanogratings with ridge width up to $750 \mathrm{~nm}$. The angular modulation of FA maturation is only partially retained on nanograting with ridge width of $1000 \mathrm{~nm}$, whereas it is almost completely lost on nanogratings with ridge width equal to or larger than $1250 \mathrm{~nm}$ (Figure 4a).

We finally assess the overall degree of FA maturation on all the tested substrates (Figure 4b). To this end, we categorize FAs according to their size into small (smaller than $1 \mu \mathrm{m}^{2}$, corresponding to focal complexes), intermediate (between 1 and $2 \mu \mathrm{m}^{2}$ ), and large (larger than $2 \mu \mathrm{m}^{2}$ ). PC12 cells differentiating on all the tested nanogratings produce a similar fraction of small FAs, indicating that the process of adhesion initiation is not affected by the ridge width in the tested range (Figure $4 \mathrm{~b}$ ).
Interestingly, the fraction of large FAs is slightly decreased on nanogratings with large ridge width (D, E, and F, Figure 4b), where the fraction of intermediate FAs increases correspondingly.

Effect of Nanogratings with Increasing Ridge Width on Neurite Pathfinding and Neuronal Polarity Establishment. In order to quantify the effect of the different nanogratings on neuronal polarity establishment and neurite pathfinding, we measure the length, alignment to the nanogratings, and the number of neurites generated by PC12 cells after 4 days of NGF stimulation (Figure 5). Figure 5a displays the average length of neurites produced by $\mathrm{PC} 12$ cells on nanogratings with increasing ridge width. The length of neurites produced on nanogratings with 
a

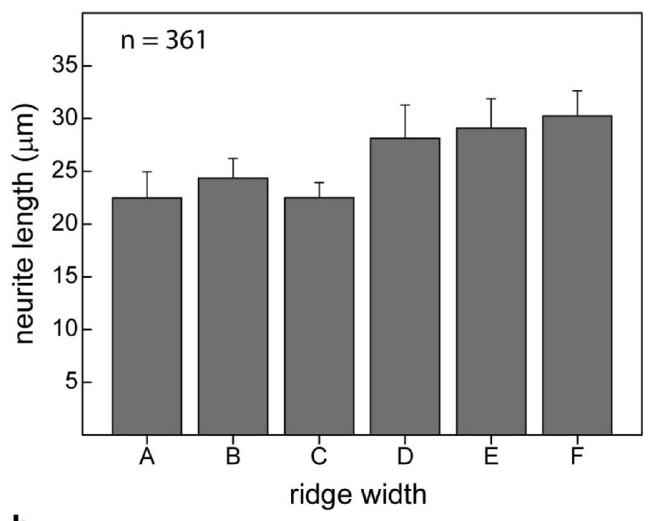

b

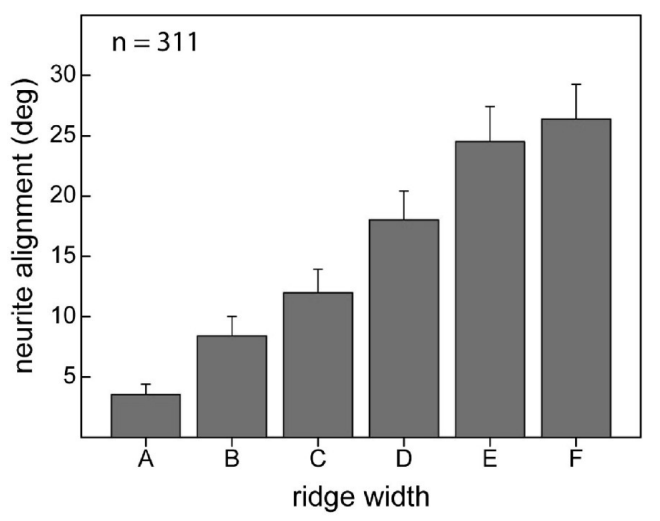

C

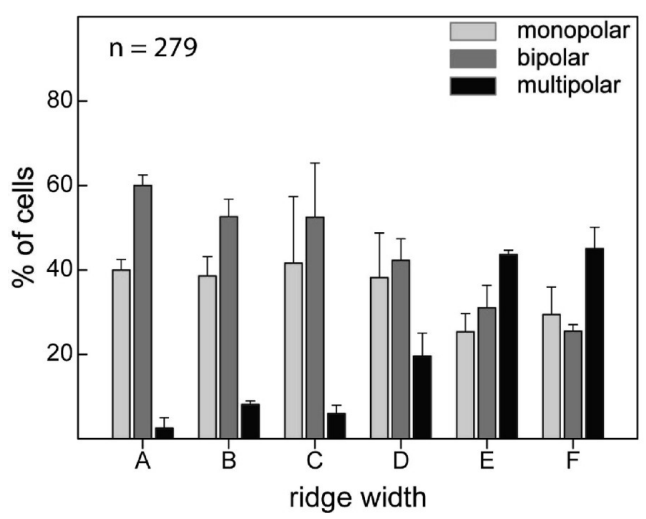

Figure 5. Neurite pathfinding and neuronal polarity establishment during PC12 differentiation on nanogratings with increasing ridge width. (a) Neurite alignment to nanogratings $96 \mathrm{~h}$ after NGF stimulation of PC12 cells. The graph reports the mean neurite alignment (in degrees) on nanogratings with ridge width corresponding to 500, 750, 1000, 1250, 1500, or $2000 \mathrm{~nm}$ (nanograting A, B, C, D, E, and F, respectively). (b) Length of neurites generated by $\mathrm{PC} 12$ cells differentiating on nanogratings with increasing ridge width. The graph reports the average measured length of neurites generated by differentiating PC12 cells after $96 \mathrm{~h}$ of stimulation with NGF. (c) Polarity of differentiated PC12 cells on nanogratings. The graph reports the percentage of mono-, bi-, and multipolar cells (light gray, gray, and black histograms, respectively) after $96 \mathrm{~h}$ of NGF stimulation on nanogratings. The number of analyzed elements is reported in the upper left corner of the graphs. Error bars correspond to the measured standard error of the mean. Detailed statistical comparison between the reported measurements is provided in Supplementary Figure 2 (Supporting Information).

ridge width ranging from 500 to $1500 \mathrm{~nm}(\mathrm{~A}-\mathrm{E})$ is not significantly different. In contrast, neurites generated on nanogratings with the largest ridge width (F) are significantly longer $(30.3 \pm$ $2.4 \mu \mathrm{m}$, Figure $5 \mathrm{a}$ ) than neurites generated on nanogratings with smaller ridge widths $(\mathrm{A}-\mathrm{C}$; Figure $5 \mathrm{a})$. This result reveals that neurites develop more efficiently on nanogratings with ridge width larger than the typical FA length measured on flat substrates (Supplementary Figure 1, Supporting Information).

Nanogratings with increasing ridge width have an evident impact on neurite pathfinding, measured as the average alignment angle for neurites contacting the underlying anisotropic topography (Figure $5 b$ ). The average neurite alignment after 4 days of stimulation is $3.5 \pm 0.9^{\circ}$ for cell differentiating on the smallest nanogratings tested (A). The average neurite alignment increases with the increasing ridge width (Figure $5 \mathrm{~b}$ ) being $8.4 \pm$ $1.6^{\circ}, 12.0 \pm 2.0^{\circ}, 18.1 \pm 2.4^{\circ}$, and $24.5 \pm 3.0^{\circ}$ for nanogratings with ridge width $750,1000,1250$, and $1500 \mathrm{~nm}$, respectively. Neurite alignment on nanogratings with larger ridge width (2000 $\mathrm{nm}, \mathrm{F}$ ) reaches a plateau value of $26.4 \pm 2.9^{\circ}$. Altogether these results demonstrate that neurite pathfinding in differentiating PC12 cells can be modulated by nanogratings with ridge width ranging between 500 and $1500 \mathrm{~nm}$, thus exactly overlapping the typical size of FAs formed at the tip of developing neurites. Since a residual alignment is still present in nanogratings with large ridge width (average alignment of neurites developed on flat substrate is $46.5 \pm 7.1^{\circ}$ ), besides the modulation of FAs maturation an additional mechanism has to be implied into neurite pathfinding on nanogratings.

Neuronal polarity selection and neurite pathfinding are closely entangled on nanogratings inducing efficient alignment of developing neurites. ${ }^{10}$ Figure $5 \mathrm{c}$ depicts the polarity distribution after 4 days of stimulation for PC12 cells contacting nanogratings with increasing ridge width. Nanogratings with ridges smaller or equal to $1000 \mathrm{~nm}$ select bipolar cells $(60.0 \pm 2.5 \%, 52.6 \pm 4.2 \%$, and $52.5 \pm 12.8 \%$ on nanogratings $\mathrm{A}, \mathrm{B}$, and $\mathrm{C}$ respectively). Consistently, multipolar cells are almost completely absent on these substrates $(2.5 \pm 1 \%, 8.1 \pm 0.8 \%$, and $5.9 \pm 2.0 \%$ on nanogratings $\mathrm{A}, \mathrm{B}$, and $\mathrm{C}$, respectively). The polarity selection mechanism populating the fraction of bipolar cells is significantly less efficient on nanogratings with ridge width of $1250 \mathrm{~nm}$ (Figure 5c). On this substrate, $42.3 \pm 5.1 \%$ of differentiated cells is bipolar whereas $19.6 \pm 5.5 \%$ is multipolar. Even more strikingly, multipolar cells are the majority of differentiated cells on nanogratings with ridge widths of 1500 and $2000 \mathrm{~nm}$ (43.7 \pm $1.0 \%$ and $45.1 \pm 5.0 \%$, respectively). Consistently the selection of bipolar cells is lost on these substrates (30.9 $\pm 5.3 \%$ and $25.5 \pm 1.5 \%)$ and is similar to findings on flat substrates $(35.0 \pm$ $9.0 \%$ of bipolar cells and $34.5 \pm 2.1 \%$ of multipolar cells). Altogether, these data demonstrate that neuronal polarity establishment is critically modulated by the ridge width of the underlying nanogratings, with a significant step at $1250 \mathrm{~nm}$.

The described effects on neurite pathfinding and neuronal polarity establishment are summarized in Figure 6. After 4 days of NGF stimulation, PC12 contacting nanogratings with the smallest ridge width $(500 \mathrm{~nm})$ are induced to produce two long neurites highly aligned to the underlying topography (Figure 6a). Neurite alignment decreases in nanogratings with increasing ridge width (750-1250 nm, Figure 6d) and highly misaligned processes are formed by stimulated PC12 cells on nanogratings with ridge width larger than $1500 \mathrm{~nm}$ (Figure 6b). Thus, neurite pathfinding is modulated on nanogratings with increasing ridge width, with the best alignment obtained on nanogratings with ridge width smaller than $1000 \mathrm{~nm}$. A similar effect is observed on neuronal polarity. Nanogratings with small ridge width (smaller 


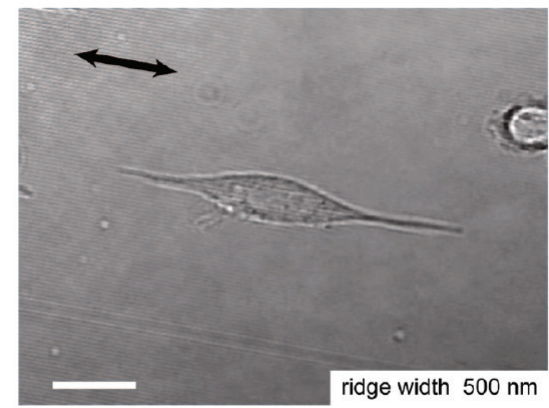

c

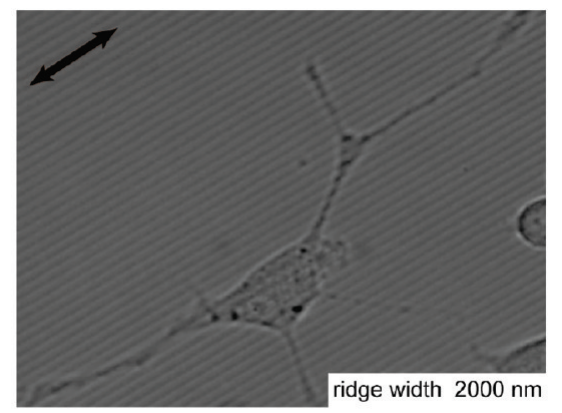

b

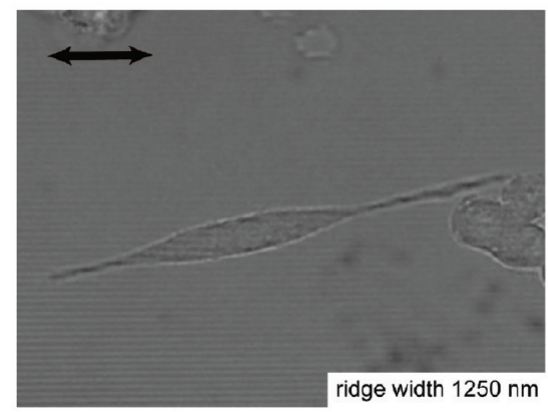

d

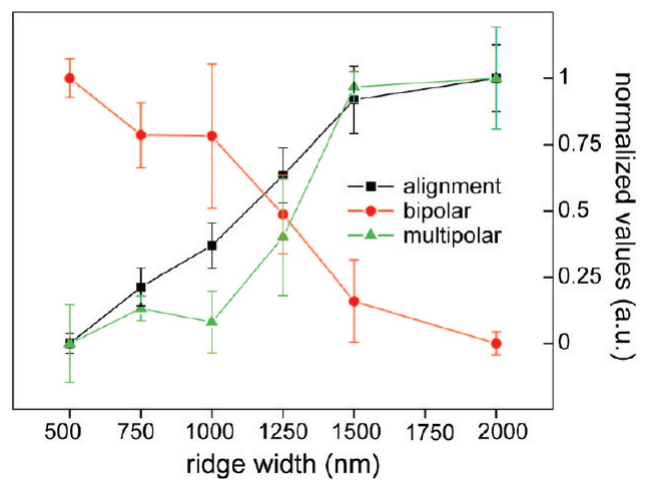

Figure 6. Modulation of neuronal polarity selection by nanogratings. Nanogratings with increasing ridge width select alternatively (a) bipolar cells with two aligned neurites on nanograting A (ridge width $500 \mathrm{~nm}$ ), (b) bipolar cells with misaligned neurites on nanograting D (ridge width $1250 \mathrm{~nm}$ ), (c) or multipolar cells with misaligned neurites on nanograting F (ridge width $2000 \mathrm{~nm}$ ). Scale bar in panel a corresponds to $20 \mu \mathrm{m}$. Black arrows in each panel indicate the direction of the nanograting. The corresponding ridge width is reported in the lower left corner. (d) Normalized neurite alignment (black line connecting full black squares), normalized percentage of bipolar cells (red line connecting full red circles), and normalized percentage of multipolar cells (green line connecting green triangles), on nanogratings with increasing ridge width. Error bars correspond to the measured standard error of the mean.

than $1250 \mathrm{~nm}$, Figure 6d) select bipolar cells, while multipolar cells are almost absent. A large fraction of multipolar cells is instead visible on nanogratings with large ridge width $(2000 \mathrm{~nm}$, Figure 6c) indicating that a different neuronal polarity selection mechanism operates on these topographies. Additionally, this analysis demonstrates that the mechanisms regulating neurite pathfinding and neuronal polarity selection on nanogratings are sensitive to different ridge width ranges. While neurite pathfinding is modulated by ridge widths ranging from 500 to $1500 \mathrm{~nm}$ with maximal sensitivity between 750 and $1250 \mathrm{~nm}$ (Figure 6d), the mechanism selecting neuronal polarity on nanogratings tolerates ridge width changes in the range between 500 and $1000 \mathrm{~nm}$ while is critically sensitive to changes in the range between 1000 and $1500 \mathrm{~nm}$. Altogether these results indicate that the transition from bipolar cells with aligned neurites to multipolar cells with misaligned neurites (Figure 6) goes along with an increase in the number and size of misaligned FAs (Figures 3 and 4), suggesting that the mechanisms responsible for polarity selection gradually reduced their efficiency when the topographical constraint on FA maturation is released.

Our approach exploits gratings with submicrometer featuresize with the following rationale: the groove size and depth are chosen to ensure FA confinement onto ridges. Individual FAs cannot bridge over physical or chemical barriers wider than $500 \mathrm{~nm}^{10,29}$ while cells show maximal contact guidance when grooves are deeper than $350 \mathrm{~nm}^{30}$ Thus, the FA establishment and maturation are influenced by a single linear parameter, i.e., the ridge width (Figures 3 and 4). This is varied over a range encompassing the typical size of adhesions established by PC12 cells. As expected, nanogratings with ridge width smaller than the typical length of FAs (from 500 to $1500 \mathrm{~nm}$ ) apply a topographical constraint leading to adhesion maturation only at the tip of aligned neurites while forcing the collapse of adhesions at the tip of misaligned ones (Figures 1 and 2). This yields a fine modulation of neurite pathfinding (Figure 5) and demonstrates that between 500 and $1500 \mathrm{~nm}$ the average neurite alignment can be controlled as a function of the lateral ridge sizes with an accuracy of $0.20 \pm 0.02^{\circ}$ over $10 \mathrm{~nm}$.

Nanogratings with increasing ridge width also allow control over the average number of neurites per differentiating neuron (Figures 5 and 6). Nanogratings with the smallest ridge size (500 $\mathrm{nm}$ ) strongly favor bipolar cells (Figure 5). The mechanism selecting bipolar cells is tolerant in the ridge-width range between 500 and $1000 \mathrm{~nm}$. A transition to multipolarity is obtained increasing the ridge width to $1500 \mathrm{~nm}$ (Figure 5). This result demonstrates that neurite pathfinding and neuronal polarity establishment can be decoupled by pure topographical means. The mechanisms controlling these processes are differently sensitive to ridge-width changes when contacting nanogratings (Figures 5 and 6). Neurite pathfinding is responsive to ridge-width variations between 500 and $1000 \mathrm{~nm}$. Differently, the mechanism controlling neuronal polarity establishment senses variation in ridge width between 1000 and $1500 \mathrm{~nm}$.

In conclusion, we have designed and fabricated a set of biocompatible textured scaffolds selectively favoring specific neuronal polarity states. We have demonstrated that the selection 
mechanism can be tailored by modulating the topographical constraint applied to the maturation of focal adhesions during neuritogenesis. This approach allows an independent control over the orientation and number of neurites produced by differentiating cells and may be applied to other biological systems where cell orientation and polarity are essential to the tissue functionality.

\section{ASSOCIATED CONTENT}

S Supporting Information. Detailed information on materials and methods and supplementary figures. This material is available free of charge via the Internet at http://pubs.acs.org.

\section{AUTHOR INFORMATION}

\section{Corresponding Author}

*E-mail: Aldo Ferrari, aferrari@ethz.ch; Marco Cecchini, m.cecchini@sns.it.

\section{Present Addresses}

${ }^{\perp}$ Laboratory of Thermodynamics in Emerging Technologies, ETH Zurich, Sonneggstrasse 3, CH-8092 Zurich, Switzerland.

\section{ACKNOWLEDGMENT}

The authors are grateful for the support of the Italian Institute of Technology. This work was supported partly by MIUR under FIRB Projects No. RBLA03ER38 and RBIN045MNB and partly by the European Commission through the European Collaborative Projects NANOCARD and NANOII within FP7. D.P. also thanks the Apulia Strategic Regional Project PS_144. We thank Dr. Francesca Signori for technical support.

\section{REFERENCES}

(1) Arimura, N.; Kaibuchi, K. Nat. Rev. Neurosci. 2007, 8 (3), $194-$ 205.

(2) Tessier Lavigne, M.; Goodman, C. S. Science 1996, 274 (5290), $1123-1133$.

(3) Woo, S.; Gomez, T. M. J. Neurosci. 2006, 26 (5), 1418-1428.

(4) Bentley, D.; Toroian-Raymond, A. Nature 1986, 323 (6090), $712-5$.

(5) Kulkarni, R. P.; Bak-Maier, M.; Fraser, S. E. Proc. Natl. Acad. Sci. U.S.A. 2007, 104 (4), 1207-12.

(6) Geiger, B.; Bershadsky, A.; Pankov, R.; Yamada, K. M. Nat. Rev. Mol. Cell Biol. 2001, 2 (11), 793-805.

(7) Balaban, N. Q.; Schwarz, U. S.; Riveline, D.; Goichberg, P.; Tzur, G.; Sabanay, I.; Mahalu, D.; Safran, S.; Bershadsky, A.; Addadi, L.; Geiger, B. Nat. Cell Biol. 2001, 3 (5), 466-72.

(8) Bershadsky, A. D.; Ballestrem, C.; Carramusa, L.; Zilberman, Y.; Gilquin, B.; Khochbin, S.; Alexandrova, A. Y.; Verkhovsky, A. B.; Shemesh, T.; Kozlov, M. M. Eur. J. Cell Biol. 2006, 85 (3-4), 165-73.

(9) Arnold, M.; Hirschfeld-Warneken, V. C.; Lohmuller, T.; Heil, P.; Blummel, J.; Cavalcanti-Adam, E. A.; Lopez-Garcia, M.; Walther, P.; Kessler, H.; Geiger, B.; Spatz, J. P. Nano Lett. 2008, 8 (7), 2063-2069.

(10) Ferrari, A.; Cecchini, M.; Serresi, M.; Faraci, P.; Pisignano, D.; Beltram, F. Biomaterials 2010, 31 (17), 4682-4694.

(11) Huang, J. H.; Grater, S. V.; Corbellinl, F.; Rinck, S.; Bock, E.; Kemkemer, R.; Kessler, H.; Ding, J. D.; Spatz, J. P. Nano Lett. 2009, 9 (3), 1111-1116.

(12) Geiger, B.; Spatz, J. P.; Bershadsky, A. D. Nat. Rev. Mol. Cell Biol. 2009, 10 (1), 21-33.

(13) Steinberg, T.; Schulz, S.; Spatz, J. P.; Grabe, N.; Mussig, E.; Kohl, A.; Komposch, G.; Tomakidi, P. Nano Lett. 2007, 7 (2), 287-94.

(14) Ferrari, A.; Cecchini, M.; Degl Innocenti, R.; Beltram, F. IEEE Trans. Biomed. Eng. 2009, 56 (11 Pt 2), 2692-2696.
(15) Wozniak, M. A.; Modzelewska, K.; Kwong, L.; Keely, P. J. Biochim. Biophys. Acta 2004, 1692 (2-3), 103-119.

(16) Anselme, K.; Davidson, P.; Popa, A. M.; Giazzon, M.; Liley, M.; Ploux, L. Acta Biomater. 2010, 6, 3824-3846.

(17) Bettinger, C. J.; Langer, R.; Borenstein, J. T. Angew. Chem., Int. Ed. 2009, 48 (30), 5406-15.

(18) Lee, J. Y.; Bashur, C. A.; Goldstein, A. S.; Schmidt, C. E. Biomaterials 2009, 30 (26), 4325-35.

(19) Lopez, C.A.; Fleischman, A. J.; Roy, S.; Desai, T. A. Biomaterials 2006, 27 (16), 3075-83.

(20) Foley, J. D.; Grunwald, E. W.; Nealey, P. F.; Murphy, C. J. Biomaterials 2005, 26 (17), 3639-44.

(21) Patel, S.; Kurpinski, K.; Quigley, R.; Gao, H.; Hsiao, B. S.; Poo, M. M.; Li, S. Nano Lett. 2007, 7 (7), 2122-8.

(22) Yim, E. K.; Pang, S. W.; Leong, K. W. Exp. Cell Res. 2007, 313 (9), 1820-9.

(23) Cecchini, M.; Bumma, G.; Serresi, M.; Beltram, F. Nanotechnology 2007, 18 (50), No. 505103.

(24) Engler, A. J.; Sen, S.; Sweeney, H. L.; Discher, D. E. Cell 2006, 126 (4), 677-89.

(25) Tzvetkova-Chevolleau, T.; Stephanou, A.; Fuard, D.; Ohayon, J.; Schiavone, P.; Tracqui, P. Biomaterials 2008, 29 (10), 1541-51.

(26) Lamoureux, P.; Buxbaum, R. E.; Heidemann, S. R. Nature 1989, 340 (6229), 156-162.

(27) Ferrari, A.; Faraci, P.; Cecchini, M.; Beltram, F. Biomaterials 2010, 31, 2565-73.

(28) Huang, C.; Borchers, C. H.; Schaller, M. D.; Jacobson, K. J. Cell Biol. 2004, 164 (4), 593-602.

(29) Arnold, M.; Schwieder, M.; Blummel, J.; Cavalcanti-Adam, E. A.; Lopez-Garcia, M.; Kessler, H.; Geiger, B.; Spatz, J. P. Soft Matter 2009, 5 (1), 72-77.

(30) Loesberg, W. A.; te Riet, J.; van Delft, F. C. M. J. M.; Schon, P.; Figdor, C. G.; Speller, S.; van Loon, J. J. W. A.; Walboomers, X. F.; Jansen, J. A. Biomaterials 2007, 28 (27), 3944-3951. 